\title{
Highly efficient algorithms for CIS type excited state wave function overlaps
}

\author{
Marin Sapunar, ${ }^{*}$ Tomislav Piteša, Davor Davidović, and Nađa Došlić \\ Department of Physical Chemistry, Ruđer Bošković Institute, Zagreb
}

E-mail: marin.sapunar@irb.hr

\begin{abstract}
Two algorithms for calculating overlaps between CIS (or TDDFT) type excited state wave functions are presented, one based on expansion of overlap determinants into level 2 minors (OL2M) and the other based on an expansion of the wave functions into natural transition orbitals (ONTO). Both are significantly faster than previously available algorithms, with the ONTO algorithm reducing the cost of a single overlap element calculation by a factor of the square of the number of occupied orbitals in the system, resulting in orders of magnitude faster calculations for large systems and significantly increasing the size of systems for which TDDFT based nonadiabatic dynamics simulations can be performed. The OL2M algorithm is significantly slower for a single overlap matrix element, but becomes preferred when overlaps between large numbers of states are required. Additionally, we test the accuracy of approximate overlaps calculated using truncated wave functions and show that truncation can lead to very large errors in the overlaps. Lastly, we provide examples of applications for wave function overlaps outside the context of nonadiabatic dynamics.
\end{abstract}




\section{Introduction}

Overlaps between many-electron wave functions play an important role in photochemical studies as they offer an intuitive and straightforward way to track the electronic character of the states along different nuclear geometries. As such, they are often used for constructing multi-state, multi-dimensional potential energy surfaces (PES) for quantum dynamics. ${ }^{1-15}$ In photoionization, the computation of transition dipole matrix elements using the Dyson orbital approach requires the computation of overlaps between the initially excited state and the final ionic bound states. ${ }^{16-21}$

Overlaps are also extensively used in the context of nonadiabatic dynamics simulations. Initially, they were introduced as a means of approximating time-derivative couplings $\left\langle\Psi^{A}\left|\frac{\partial}{\partial t}\right| \Psi^{B}\right\rangle$ (TDCs) in fewest-switches surface hopping (FSSH) ${ }^{22,23}$ calculations. This was primarily done in situations when an analytic calculation of nonadiabatic coupling vectors was not available or too expensive, ${ }^{23,24}$ and was shown to give results in agreement with the analytic method. ${ }^{24,25}$ More recently, a number of methods, ${ }^{24,26-30}$ all employing wave function overlaps, have been developed to address the trivial crossings problem in nonadiabatic dynamics. This problem occurs at intersections between weakly coupled states, where nonadiabatic couplings are highly peaked and the entire region where the coupling is significant can be jumped over in a single time step. In such cases the dynamics proceed in the same adiabatic state when a hop is supposed to occur for the system to remain in the same diabatic state. These problems are more pronounced in large molecules or clusters of molecules where couplings between states are more often vanishingly small due to the spatial separation between the excitations for different states. ${ }^{27,31,32}$ Peaks in the couplings can be avoided altogether by switching to a locally diabatic basis. ${ }^{26}$ When propagation in the adiabatic representation is preferred, sudden changes in the electronic structure of the adiabatic states (crossings between diabatic states) can be detected from the overlap matrix elements and the trajectory can be forced to follow the correct diabatic state ${ }^{24,27-29}$ or the overlap matrix can be interpolated while preserving the norm of the states ${ }^{30}$ which recovers 
the peak in the couplings at state crossings. These corrections allow larger time steps to be used in dynamics calculations, significantly reducing their overall cost.

A major limitation of wave function overlap based approaches is their unfavourable scaling with the size of the system. Full overlap calculations are always slower than the orbital derivatives method for approximating $\mathrm{TDCs}^{33}$ and can become more expensive than the analytic calculation of nonadiabatic coupling vectors (and the entire electronic structure calculation) for larger systems. Since (at least approximate) overlaps are needed to treat trivial crossings, speeding up the calculation of overlaps can significantly increase the size of systems for which FSSH simulations can be reliably performed.

In this work, we present two highly efficient algorithms for calculating overlaps between excited states which can be written using CIS type wave functions. This covers excited states obtained by two electronic structure methods frequently used in FSSH calculations, linear response time-dependent density functional theory (LR-TDDFT) and the algebraic diagrammatic construction to second order $(\operatorname{ADC}(2)) .{ }^{34,35}$ For LR-TDDFT, the Casida density assignment ansatz ${ }^{36}$ is used to construct excited state auxiliary wave functions as a sum of singly excited Slater determinants constructed from Kohn-Sham orbitals. ${ }^{37,38}$ For ADC(2) and other correlated single reference methods, exact wave function overlaps are usually not available, but approximate overlaps can be obtained if single excitations are assumed to be the dominant contribution. ${ }^{39,40}$

Furthermore, we show how an overlap-based assignment procedure for following the character of the electronic states at different geometries ${ }^{27}$ can be used to simplify common tasks in photochemical studies such as locating minima on excited state PES and assigning spectra. 


\section{Theory}

\subsection{Overview}

We are interested in calculating the overlap matrix between a set of $N_{A}$ electronic states $\left\{\left|\Psi_{A}\right\rangle\right\}$ and $N_{B}$ states $\left\{\left|\Psi_{B}^{\prime}\right\rangle\right\}$ with elements

$$
S_{A B}=\left\langle\Psi_{A} \mid \Psi_{B}^{\prime}\right\rangle
$$

In general, the only requirement is that the states have the same number of electrons of each spin. Additionally, we limit our scope to states described by CIS type wave functions

$$
\begin{aligned}
\left|\Psi_{A}\right\rangle & =\sum_{\sigma}^{\{\alpha, \beta\}} \sum_{a}^{n_{\sigma}} \sum_{i}^{m_{\sigma}} d_{a i, \sigma}^{A}\left|\Phi_{a, \sigma}^{i}\right\rangle \\
\left|\Psi_{B}^{\prime}\right\rangle & =\sum_{\tau}^{\{\alpha, \beta\}} \sum_{b}^{n_{\tau}} \sum_{j}^{m_{\tau}^{\prime}} d_{b j, \tau}^{\prime B}\left|\Phi_{b, \tau}^{\prime j}\right\rangle,
\end{aligned}
$$

where $n_{\sigma}$ and $m_{\sigma}$ are, respectively, the number of occupied and virtual orbitals of spin $\sigma$, $\left|\Phi_{a, \sigma}^{i}\right\rangle$ are Slater determinants (SDs) with a single excitation from canonical occupied orbital $a$ to virtual orbital $i$ of $\operatorname{spin} \sigma$ with CI coefficients $d_{a i, \sigma}^{A}$. Primes denote that the states in $\left\{\left|\Psi_{B}^{\prime}\right\rangle\right\}$ can differ from those in $\left\{\left|\Psi_{A}\right\rangle\right\}$ in the CI coefficients, MO coefficients, molecular geometry or basis set.

Before considering the overlaps of CIS wave functions, it is informative to consider the overlap of two SDs. It can be shown that this is equal to the determinant of the matrix of overlaps between the (HF or KS) orbitals composing the two SDs. ${ }^{41,42}$ Since overlaps for orbitals of different spin vanish, this matrix is block diagonal which allows solving the $\alpha$ and 
$\beta$ parts separately. The overlap of the two reference determinants is

$$
\begin{aligned}
\left\langle\Phi_{0} \mid \Phi_{0}^{\prime}\right\rangle & =\left|\begin{array}{ccc}
\left\langle\phi_{1 \alpha} \mid \phi_{1 \alpha}^{\prime}\right\rangle & \cdots & \left\langle\phi_{1 \alpha} \mid \phi_{n_{\alpha} \alpha}^{\prime}\right\rangle \\
\vdots & \ddots & \vdots \\
\left\langle\phi_{n_{\alpha} \alpha} \mid \phi_{1 \alpha}^{\prime}\right\rangle & \cdots & \left\langle\phi_{n_{\alpha} \alpha} \mid \phi_{n_{\alpha} \alpha}^{\prime}\right\rangle
\end{array}\right| \times\left|\begin{array}{ccc}
\left\langle\phi_{1 \beta} \mid \phi_{1 \beta}^{\prime}\right\rangle & \cdots & \left\langle\phi_{1 \beta} \mid \phi_{n_{\beta} \beta}^{\prime}\right\rangle \\
\vdots & \ddots & \vdots \\
\left\langle\phi_{n_{\beta} \beta} \mid \phi_{1 \beta}^{\prime}\right\rangle & \cdots & \left\langle\phi_{n_{\beta} \beta} \mid \phi_{n_{\beta} \beta}^{\prime}\right\rangle
\end{array}\right| \\
& =\left\langle\Phi_{0} \mid \Phi_{0}^{\prime}\right\rangle_{\alpha}\left\langle\Phi_{0} \mid \Phi_{0}^{\prime}\right\rangle_{\beta},
\end{aligned}
$$

where we define $\left\langle\Phi_{0} \mid \Phi_{0}^{\prime}\right\rangle_{\sigma}$ as the determinant of the spin $\sigma$ block of the overlap.

Inserting the CIS wave functions 2 and 3 into Eq. 1 we get

$$
\left\langle\Psi_{A} \mid \Psi_{B}^{\prime}\right\rangle=\sum_{\sigma}^{\{\alpha, \beta\}} \sum_{\tau}^{\{\alpha, \beta\}} \sum_{a}^{n_{\sigma}} \sum_{b}^{n_{\tau}} \sum_{i}^{m_{\sigma}} \sum_{j}^{m_{\tau}^{\prime}} d_{a i, \sigma}^{A} d_{b j, \tau}^{\prime B}\left\langle\Phi_{a, \sigma}^{i} \mid \Phi_{b, \tau}^{\prime j}\right\rangle
$$

and after splitting the SD overlaps into blocks as in Eq. 4 and rearranging the sums

$$
\begin{aligned}
\left\langle\Psi_{A} \mid \Psi_{B}^{\prime}\right\rangle & =\left\langle\Phi_{0} \mid \Phi_{0}^{\prime}\right\rangle_{\beta}\left(\sum_{a}^{n_{\alpha}} \sum_{b}^{n_{\alpha}} \sum_{i}^{m_{\alpha}} \sum_{j}^{m_{\alpha}^{\prime}} d_{a i, \alpha}^{A} d_{b j, \alpha}^{\prime B}\left\langle\Phi_{a}^{i} \mid \Phi_{b}^{\prime j}\right\rangle_{\alpha}\right) \\
& +\left(\sum_{a}^{n_{\alpha}} \sum_{i}^{m_{\alpha}} d_{a i, \alpha}^{A}\left\langle\Phi_{a}^{i} \mid \Phi_{0}^{\prime}\right\rangle_{\alpha}\right)\left(\sum_{b}^{n_{\beta}} \sum_{j}^{m_{\beta}^{\prime}} d_{b j, \beta}^{\prime B}\left\langle\Phi_{0} \mid \Phi_{b}^{\prime j}\right\rangle_{\beta}\right) \\
& +\left(\sum_{a}^{n_{\beta}} \sum_{i}^{m_{\beta}} d_{a i, \beta}^{A}\left\langle\Phi_{a}^{i} \mid \Phi_{0}^{\prime}\right\rangle_{\beta}\right)\left(\sum_{b}^{n_{\alpha}} \sum_{j}^{m_{\alpha}^{\prime}} d_{b j, \alpha}^{\prime B}\left\langle\Phi_{0} \mid \Phi_{b}^{\prime j}\right\rangle_{\alpha}\right) \\
& +\left\langle\Phi_{0} \mid \Phi_{0}^{\prime}\right\rangle_{\alpha}\left(\sum_{a}^{n_{\beta}} \sum_{b}^{n_{\beta}} \sum_{i}^{m_{\beta}} \sum_{j}^{m_{\beta}^{\prime}} d_{a i, \beta}^{A} d_{b j, \beta}^{\prime B}\left\langle\Phi_{a}^{i} \mid \Phi_{b}^{\prime j}\right\rangle_{\beta}\right)
\end{aligned}
$$

The above equation consists of eight blocks (four for each spin) which can be calculated separately. The most expensive step is the calculation of $n_{\sigma}^{2} m_{\sigma} m_{\sigma}^{\prime}$ determinants of the form $\left\langle\Phi_{a}^{i} \mid \Phi_{b}^{\prime j}\right\rangle_{\sigma}$. Since the calculation of a determinant is itself an $O\left(n_{\sigma}^{3}\right)$ calculation, the overall scaling for this block is $O\left(n_{\sigma}^{5} m_{\sigma} m_{\sigma}^{\prime}\right)$ and this quickly becomes the bottleneck of the entire calculation. Apart from the determinant calculation, the double sums themselves can be a 
costly step since they have to be computed for each pair of states as the CI coefficients in the sums are state dependent. The algorithm for calculating overlaps based on equation 6 will be denoted OCIS further in the text.

Plasser and coworkers ${ }^{42}$ obtained a significant speedup for MR-CIS expansions using a single step Laplace's recursive formula during the determinant calculations. Expanding the determinants into minors along a column allows one to reuse the minors for all determinants which are different from the starting determinant by only that column. Expanding $\left\langle\Phi_{a}^{i} \mid \Phi_{b}^{\prime j}\right\rangle_{\sigma}$ along column $j$ (and dropping the index $\sigma$ for convenience) one arrives at

$$
\left\langle\Phi_{a}^{i} \mid \Phi_{b}^{\prime j}\right\rangle=\sum_{c \neq a}^{n} o_{c j}(-1)^{c+b}\left\langle\Phi_{a, c}^{i} \mid \Phi_{b}^{\prime}\right\rangle+o_{i j}(-1)^{a+b}\left\langle\Phi_{a} \mid \Phi_{b}^{\prime}\right\rangle
$$

where $o_{i j}$ denotes the overlap between molecular orbitals $i$ and $j$. Here, none of the determinants have a dependence on index $j$, meaning that the determinant calculations can be taken out of the sum over $m^{\prime}$ virtual orbitals (at a cost of an additional sum over $n$ orbitals).

\subsection{Computation of CIS wave function overlaps}

In the following we describe two separate numerical procedures aiming to reduce the cost of overlap matrix computations for CIS type wave functions. The first approach (denoted OL2M) relies on level 2 minors obtained from Laplace's recursive formula to further optimize the overlap calculation based on Eq. 6, while the second approach (denoted ONTO) makes use of the reduced number of determinants in the wave function expansions based on natural transition orbitals (NTOs). ${ }^{43-45}$

In the OL2M approach, we continue from equation 7 and further expand the resulting 
minors along the row corresponding to orbital $j$

$$
\begin{aligned}
\left\langle\Phi_{a}^{i} \mid \Phi_{b}^{\prime j}\right\rangle & =\sum_{c \neq a}^{n} \sum_{d \neq b}^{n} o_{c j} o_{i d} \operatorname{sgn}(b-d) \operatorname{sgn}(c-a)(-1)^{a+b+c+d}\left\langle\Phi_{a, c} \mid \Phi_{b, d}^{\prime}\right\rangle \\
& +o_{i j}(-1)^{a+b}\left\langle\Phi_{a} \mid \Phi_{b}^{\prime}\right\rangle
\end{aligned}
$$

where $\operatorname{sgn}(b-d)$ and $\operatorname{sgn}(c-a)$ are used because all indices beyond the removed row/column in the level 1 minors are decreased by 1 from the indices in the original matrix. Interchanging indices $a$ and $c$ or $b$ and $d$ doesn't change the minors, so there are a total of $\frac{1}{4} n^{2}(n-1)^{2}$ unique level 2 minors which need to be calculated. For CIS wave functions this fully takes the determinant calculations out of the sums over virtual orbitals. The full determinants still need to be calculated from the minors, which can be done efficiently using matrix-matrix multiplications in $O\left(n^{4} m^{\prime}+n^{3} m m^{\prime}\right)$ operations and overlaps have to be calculated from the determinants, which can be performed in $O\left(n^{2} m^{\prime} m N_{B}+n^{2} m^{\prime} N_{A} N_{B}\right)$ operations. Either the calculation of the minors, or the later steps can be the bottleneck of the calculation, depending on the system size and number of states and basis functions.

The ONTO approach is based on the expansion of the CIS wave function in terms of natural transition orbitals (NTOs) ${ }^{43-45}$

$$
\begin{aligned}
\left|\Psi_{A}\right\rangle & =\sum_{\sigma}^{\{\alpha, \beta\}} \sum_{k}^{n_{\sigma}} \lambda_{k, \sigma}^{A}\left|\Theta_{k, \sigma}^{A}\right\rangle \\
\left|\Psi_{B}\right\rangle & =\sum_{\tau}^{\{\alpha, \beta\}} \sum_{l}^{n_{\sigma}} \lambda_{l, \sigma}^{B}\left|\Theta_{l, \sigma}^{B}\right\rangle
\end{aligned}
$$

where $\left|\Theta_{k, \sigma}^{A}\right\rangle$ are singly excited SDs with excitations from the $k$ th occupied to $k$ th virtual natural orbital. In this form, the full wave function is a linear combination of only $n_{\alpha}+n_{\beta}$ Slater determinants. The natural orbitals are obtained by a singular value decomposition of the CI coefficients matrix ${ }^{44}$ which can be performed in $O\left(n_{\sigma}^{2} m_{\sigma}\right)$ operations. Inserting into 
Eq. 1 we get

$$
\begin{aligned}
\left\langle\Psi_{A} \mid \Psi_{B}^{\prime}\right\rangle & =\left\langle\Theta_{0}^{A} \mid \Theta_{0}^{\prime B}\right\rangle_{\beta}\left(\sum_{k}^{n_{\alpha}} \sum_{l}^{n_{\alpha}} \lambda_{k, \alpha}^{A} \lambda_{l, \alpha}^{\prime B}\left\langle\Theta_{k}^{A} \mid \Theta_{l}^{\prime B}\right\rangle_{\alpha}\right) \\
& +\left(\sum_{k}^{n_{\alpha}} \lambda_{k, \alpha}^{A}\left\langle\Theta_{k}^{A} \mid \Theta_{0}^{\prime B}\right\rangle_{\alpha}\right)\left(\sum_{l}^{n_{\beta}} \lambda_{l, \beta}^{\prime B}\left\langle\Theta_{0}^{A} \mid \Theta_{l}^{\prime B}\right\rangle_{\beta}\right) \\
& +\left(\sum_{k}^{n_{\beta}} \lambda_{k, \beta}^{A}\left\langle\Theta_{k}^{A} \mid \Theta_{0}^{\prime B}\right\rangle_{\beta}\right)\left(\sum_{l}^{n_{\alpha}} \lambda_{l, \alpha}^{\prime B}\left\langle\Theta_{0}^{A} \mid \Phi_{k}^{\prime B}\right\rangle_{\alpha}\right) \\
& +\left\langle\Theta_{0}^{A} \mid \Theta_{0}^{\prime B}\right\rangle_{\alpha}\left(\sum_{k}^{n_{\beta}} \sum_{l}^{n_{\beta}} \lambda_{k, \beta}^{A} \lambda_{l, \beta}^{\prime B}\left\langle\Theta_{k}^{A} \mid \Theta_{l}^{\prime B}\right\rangle_{\beta}\right)
\end{aligned}
$$

where only double sums over $n_{\sigma}$ remain, meaning only $n_{\sigma}^{2}$ determinants need to be calculated. The drawback of using Equation 11 is that the NTOs are different for each state, which means that the determinants cannot be reused when calculating overlaps between multiple states. Because of this, the scaling of the entire overlap matrix calculation using the ONTO algorithm is $O\left(N_{A} N_{B} n_{\sigma}^{5}\right)$. For typical calculations, $N_{A} N_{B} \ll n_{\sigma}^{2} \ll m_{\sigma} m_{\sigma}^{\prime}$ and the ONTO algorithm is expected to be significantly faster than the OL2M algorithm, which is expected to be significantly faster than the OL1M and OCIS algorithms.

\subsection{Wave function truncation}

For most systems of interest it is possible to calculate overlaps using the ONTO algorithm at virtually no cost compared to the electronic structure calculations. Still, it is possible to further reduce the cost of the calculation by introducing a truncation of the wave functions. ${ }^{42}$ The expansion coefficients are sorted and the $k_{t}$ largest coefficients are kept

$$
\left|\tilde{\Psi}_{A}\right\rangle=\sum_{i}^{k_{t}} \lambda_{i}^{A}\left|\Theta_{i}^{A}\right\rangle
$$


so that the norm of the truncated wave function $\left|\tilde{\Psi}_{A}\right\rangle$ is larger than a selected threshold $t$

$$
\left\langle\tilde{\Psi}_{A} \mid \tilde{\Psi}_{A}\right\rangle=\sum_{i}^{k_{t}}\left(\lambda_{i}^{A}\right)^{2} \geq t
$$

Wave functions that are dominated by a small number of terms with large coefficients will benefit most from this truncation scheme. This makes the NTO expansions ideal for this type of approximation, as expansions of Eq. 9 and 10 are typically dominated by one or two determinants. However, two problems with this approximation should be considered. First, due to loss of norm upon truncation, the overlaps are systematically underestimated unless the states are renormalized after the truncation (which can also be accomplished by orthogonalization of the overlap matrix). This was shown to give very accurate overlaps in initial tests. ${ }^{42}$ The renormalization introduces the possibility of overestimating the overlaps by a maximum of $1-t$ which is an acceptable (and expected) error. The second problem, not addressed by renormalization, is that the maximum underestimation error is $2 \sqrt{t} \sqrt{1-t}$. This can be simply illustrated with an overlap of two states dominated by the same two excitations

$$
\begin{aligned}
& \left|\Psi_{A}\right\rangle=\sqrt{t}\left|\Theta_{1}\right\rangle+\sqrt{1-t}\left|\Theta_{2}\right\rangle \\
& \left|\Psi_{B}\right\rangle=\sqrt{t}\left|\Theta_{2}\right\rangle+\sqrt{1-t}\left|\Theta_{1}\right\rangle
\end{aligned}
$$

The overlap of these two states is equal to $2 \sqrt{t} \sqrt{1-t}$ when the full wave functions are considered. If the states are truncated, only the dominant excitations are kept and the overlap is 0 . For $t=0.9$, this produces an error of 0.6 , and even for $t=0.99$ the error is still 0.2. Particularly in the case of the NTO expansion (which is often dominated by one or two excitations) the above example might be a realistic scenario which requires testing. 


\section{Results and discussion}

\subsection{Performance}

In this section we compare the performance the OL2M and ONTO algorithms with varying size of the system, basis set and number of states included. Increasing the system size has the most drastic effect on the calculation, as even the ONTO algorithm scales with the fifth power of the number of occupied orbitals. Tests were performed for a series poly-alanine peptides with 3 to 30 residues (62 to 575 occupied orbitals) employing two basis sets and 5 excited states (25 overlap matrix elements) included in the calculations. The number of virtual orbitals grows from 196 to 1843 for the small basis set (def-SV(P)) and from 656 to 6083 for the large basis set (cc-pVTZ). Timings are shown in Figure 1a for overlap matrix calculations performed on a single CPU core. In addition to results from the new algorithms, we show results using the previously available algorithm based on level 1 minors (OL1M algorithm). ${ }^{42}$ Both algorithms improve upon the OL1M algorithm, but the ONTO algorithm also outperforms the OL2M algorithm by an order of magnitude even for the smallest test case. The differences are more pronounced for larger systems. Going beyond $\approx 200$ occupied orbitals, the OL2M algorithm quickly becomes too expensive while the ONTO algorithm still takes only eight hours on a single processor at 575 occupied orbitals.

The curves for the two basis sets overlap for both algorithms, meaning that the benefits of the new algorithms are largest for calculations with large basis sets. To further show the effect of basis set size on the calculation cost, we performed calculations for the 5 Alanine system with a wider range of basis sets (Fig. 1b). The ONTO algorithm still shows almost no dependence on the basis set, as only the SVD decomposition to generate the NTOs (which is less than 0.1 second for the largest basis set) depends on the number of virtual orbitals. For the largest basis set with the ONTO algorithm more time is spent for reading the input and calculating the atomic and molecular orbital overlaps than in the wave function overlap calculation. The cost increase in the OL2M algorithm is also initially negligible. 


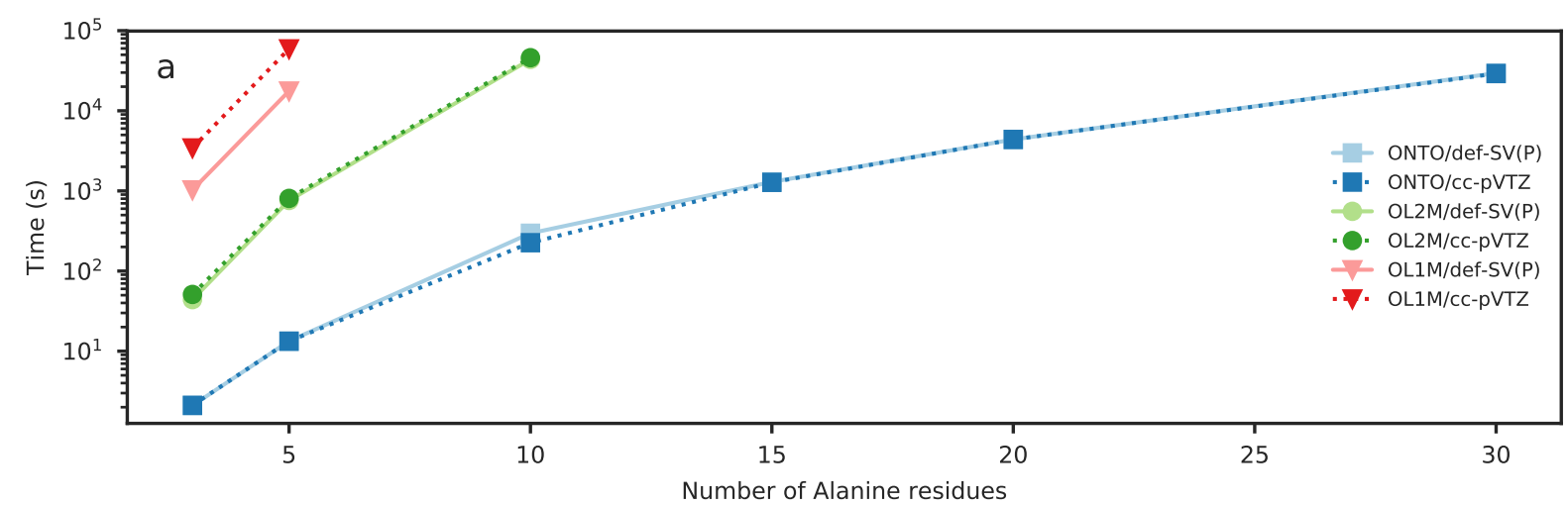

3 Alanine system
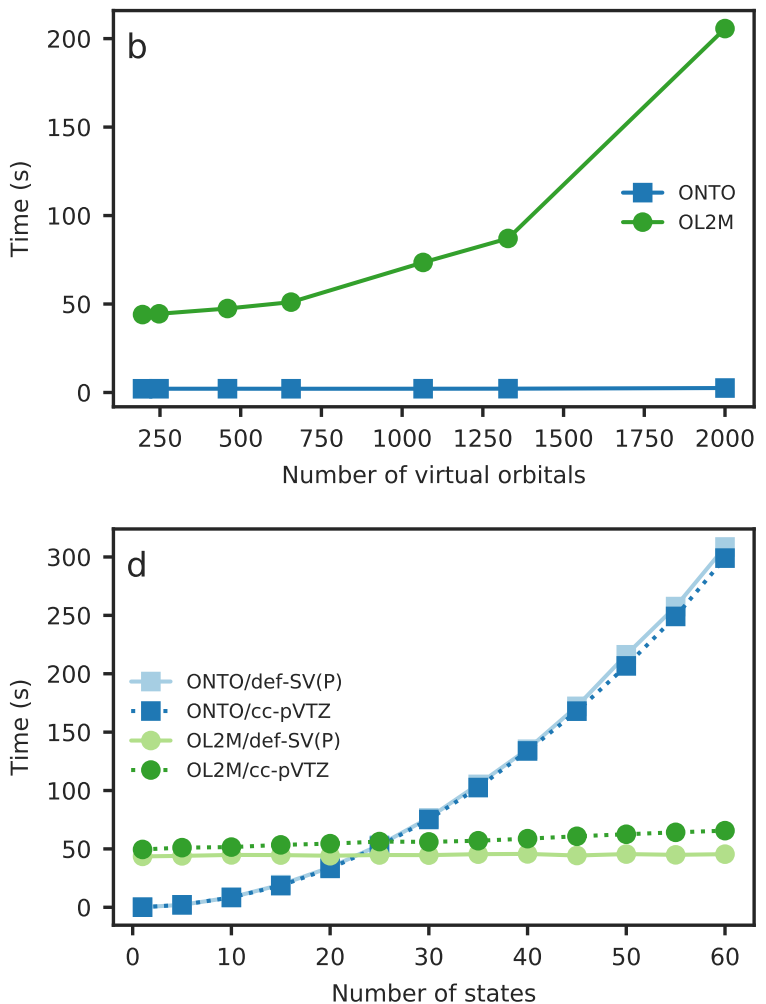

5 Alanine system
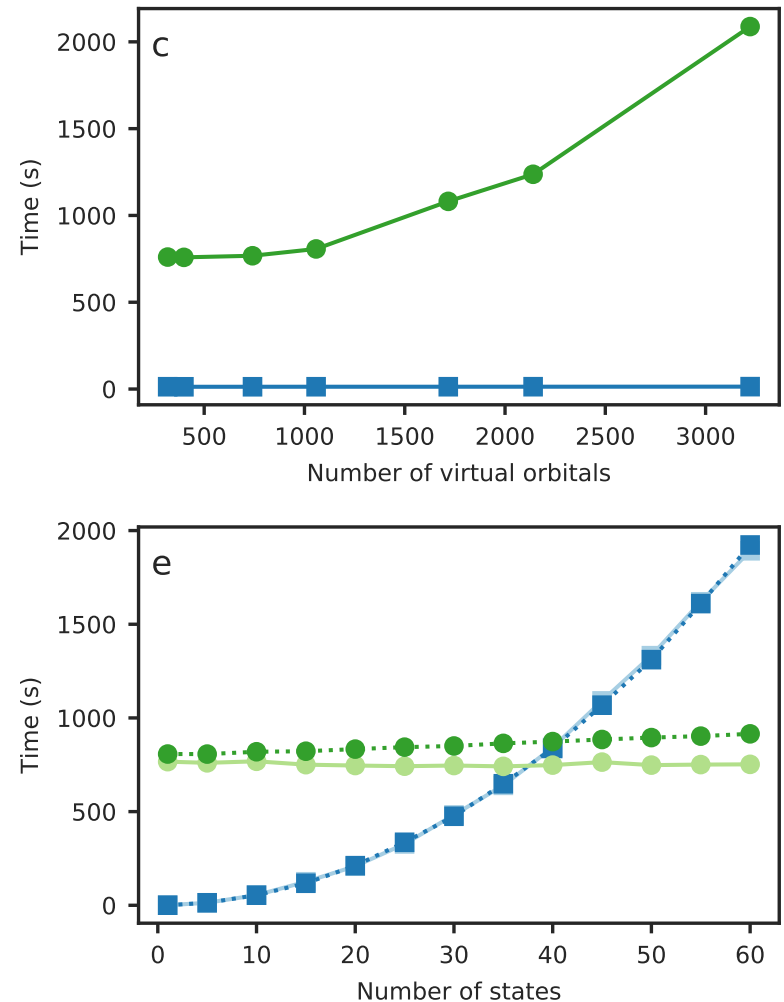

Figure 1: Comparison of the performance of the OL1M (red), OL2M (green) and ONTO (blue) algorithms. (a) Scaling with the system size (number of residues) with 5 excited states. Additional tests were performed on the 3 Alanine (b, d) and 5 Alanine (c, e) systems. (b) 3-Ala and (c) 5-Ala scaling with basis set size for 5 excited states going from the def-SV(P) to the aug-cc-pVQZ basis set. (d) 3-Ala and (e) 5-Ala scaling with the number of states included in the calculation. 
However, the cost of calculating the overlaps from the minors eventually becomes comparable to the determinant calculations and scaling with the square of the number of virtual orbitals emerges.

In a final set of tests, we look at the scaling of the two algorithms with the number of states (Fig. 1d and 1e) where we see that the OL2M algorithm becomes preferable to the NTO algorithm around 23(37) states for the 3(5) Alanine system. The dominant steps for large systems in the OL2M and ONTO algorithms are $O\left(n^{7}\right)$ and $O\left(n^{5} N_{A} N_{B}\right)$, respectively. Because of this, the OL2M algorithm becomes preferred over the ONTO algorithm when $N_{A} N_{B} / n^{2}>c$, where $c$ is some implementation dependent constant $\left(c \approx \frac{1}{6}\right.$ in our current approximation). A small increase in the cost of the OL2M algorithm is visible for the larger basis, again due to the $O\left(n^{2} m^{\prime} m N_{B}\right)$ part becoming relevant.

\subsection{Truncation}

The truncation scheme was tested for a range of different nuclear geometries along a FSSH trajectory by repeating overlap calculations using different values of the $t$ truncation threshold parameter. One TDDFT(B3LYP)/def2-TZVPD and one ADC(2)/aug-cc-pVDZ trajectory was randomly selected from a previously reported set of FSSH dynamics simulations of the pyrrole molecule excited to $\mathrm{B}_{2}\left(\pi \pi^{\star}\right)$ state. ${ }^{40}$ All electronic structure calculations were performed using the Turbomole program package. ${ }^{46-48}$ In both cases, ten excited electronic states were included in the calculations and trajectories were propagated for $250 \mathrm{fs}$ with a time step of 0.5 fs for a total of 50000 overlap matrix elements between excited states. The basis set for the TDDFT calculations included 18 occupied and 212 virtual orbitals, while the basis set for the $\mathrm{ADC}(2)$ calculation included 13 occupied (the five core orbitals were frozen) and 142 virtual orbitals. The total number of determinants computed along the trajectory is shown in Figure 2. The efficiency of the truncation scheme is determined by the number of excitations with significant coefficients in the wave function expansion. In the TDDFT calculations, the auxilliary wave functions, written in terms of excitations from 
Kohn-Sham orbitals, are dominated by only a few excitations. Because of this, for small values of $t$ the possibility of reusing determinants for multiple states in the OCIS algorithm slightly outweighs the compactness of the wave functions in the ONTO algorithm. However, as the threshold is increased above $t=0.98$, the cost of the OCIS algorithm quickly overtakes the ONTO algorithm. In the $\operatorname{ADC}(2)$ calculation, a larger number of singly excited determinants has significant contributions and the ONTO algorithm requires less determinants for $t=0.99$ than the OCIS algorithm for $t=0.9$. In both cases, the number of determinants calculated along the trajectory is lower with the ONTO algorithm by more than two orders of magnitude for higher values of $t$. For larger systems or smaller numbers of states, these differences will be even more pronounced in favour of the ONTO algorithm.
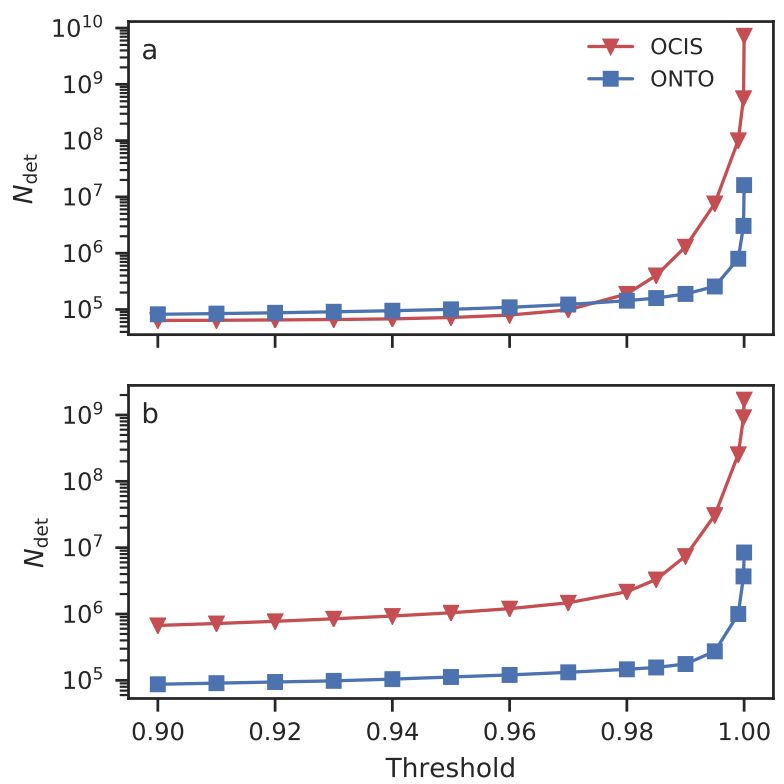

Figure 2: Comparison of the total number of determinants computed along a (a) TDDFT(B3LYP)/def2-TZVPD and (b) ADC(2)/aug-cc-pVDZ (b) FSSH trajectory of pyrrole for different values of the CI vector truncation threshold $t$ using the ONTO algorithm (blue squares) and OCIS algorithm (red circles).

The errors introduced by the truncation are shown in Figure 3. All overlap matrix elements were compared to the corresponding elements calculated using the full wave functions. The mean error of the approximation scheme is small for both the OCIS and ONTO algorithm and is reduced further by orthogonalization of the overlap matrix. However, the more 
relevant information for FSSH calculations is the maximum error, since a large error in a single matrix element can cause an unphysical hop which affects the remainder of the trajectory. Here, both algorithms perform much worse. In the case of the TDDFT trajectory, both the CIS and NTO expansions are usually dominated by a few determinants and the maximum errors are approximately the same with both algorithms. $\mathrm{ADC}(2)$ wave functions usually have contributions from a larger number of excitations. Still, the maximum errors of the OCIS algorithm are only slightly smaller than these of the ONTO algorithm. Orthogonalization has almost no effect on the maximum errors since they are not caused by the loss of norm of the wave functions. In any case, it should be assumed that, at least for TDDFT and $\operatorname{ADC}(2)$ wave functions, errors of the order $2 \sqrt{1-t}$ are possible with this approximation scheme and it should be avoided or used with the largest possible values of the truncation threshold when quantitative results are required. These results also call into question the approximate overlaps used for $\mathrm{CC} 2 / \mathrm{ADC}(2)$ calculations. Calculation of exact overlaps with these methods is not feasible even for small systems and only their singles amplitudes are taken to construct a formal CIS wave function. ${ }^{39}$ Along the tested trajectory, the mean norm of the (neglected) doubles amplitudes is 0.076 , meaning overlaps can be underestimated by as much as 0.530 even before any other approximations. 

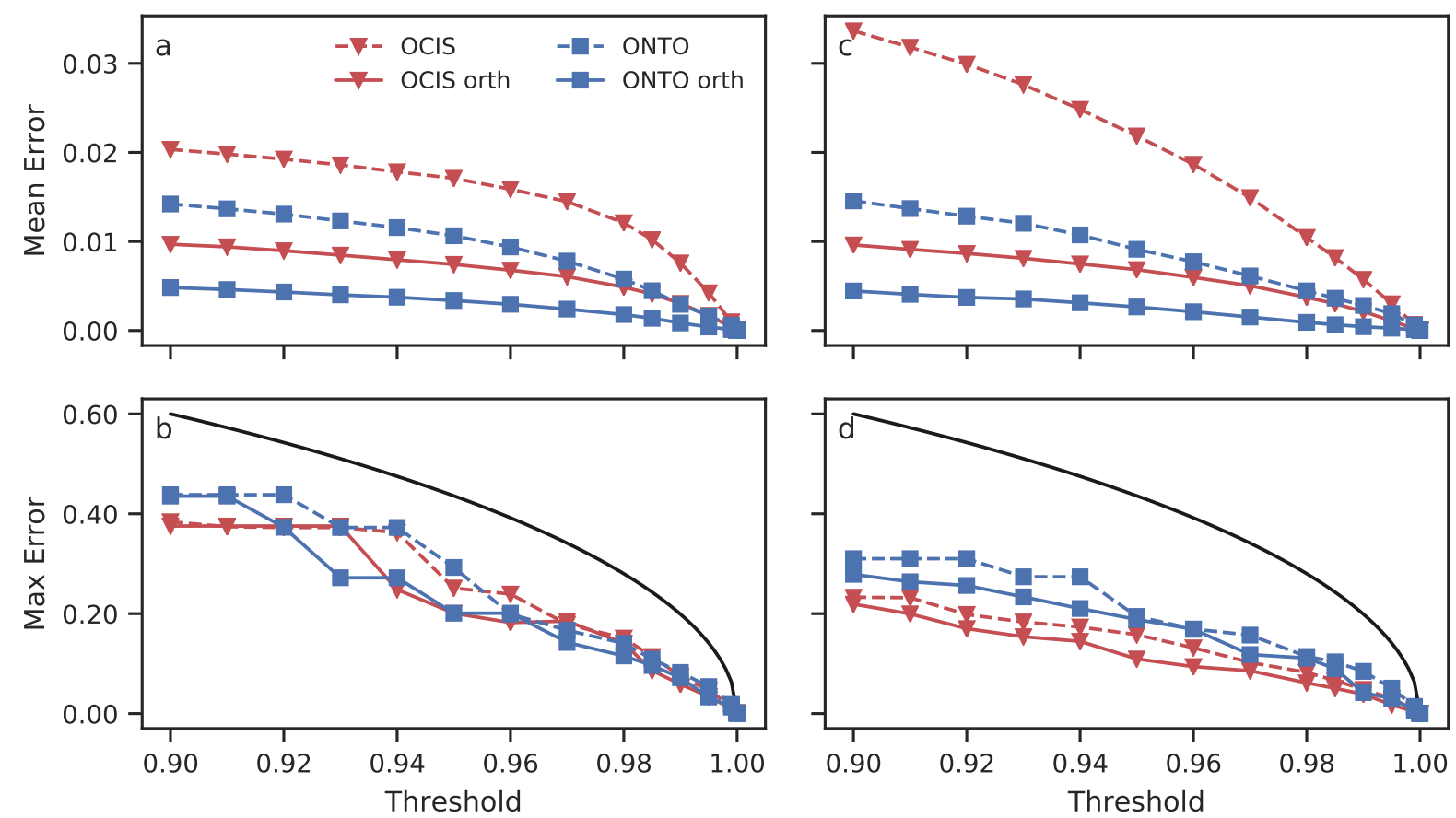

Figure 3: Mean $(\mathrm{a}, \mathrm{c})$ and maximum $(\mathrm{b}, \mathrm{d})$ errors for overlap elements computed with different threshold values along a (a, b) TDDFT(B3LYP)/def2-TZVPD and (c, d) ADC(2)/augcc-pVDZ FSSH trajectory using the ONTO algorithm (blue squares) and OCIS algorithm (red circles). Errors for the raw overlap matrix elements (full lines) and errors after orthogonalization (dashed lines) and the maximum error expected for the approximation scheme (black line) are shown. 


\subsection{Applications}

When considering excited electronic states, it is often more useful to think in terms of the electronic character of the states (diabatic states) rather than their ordering at a particular nuclear geometry (adiabatic states). However, only adiabatic states are obtained from electronic structure calculations and mapping these states at different geometries to some diabatic states defined by their electronic character is often needed in photochemical studies. This can be accomplished in a straightforward way by defining the diabatic states at a reference geometry and solving the assignment problem for the overlap matrix between the adiabatic states ${ }^{27,49}$ at the reference geometry and other geometries. In this section, we show an example of how this mapping can be used to simplify some common tasks encountered in photochemical studies. For the case study, we selected pyrrole, one of the best studied photochemical systems. ${ }^{40,50-57}$

We start with a decomposition of an absorption spectrum simulated using the nuclearensemble method ${ }^{58-60}$ into contributions by state. The absorption spectrum was calculated using a set of 8000 geometries sampled from the thermal harmonic oscillator Wigner distribution for the ground state minimum of pyrrole. The absorption spectrum is built using excitation energies and oscillator strengths calculated at these geometries at the B3LYP/def2TZVPD level. This method was used previously for simulating the first absorption band of pyrrole and was shown to be in qualitative agreement with the experimental spectrum. ${ }^{40,60}$ The intense and very narrow peak at $5.85 \mathrm{eV}$ assigned to the $\mathrm{B}_{1}\left(3 p_{y}\right)$ state, however, is not reproduced at with this method. To decompose the spectrum, adiabatic states at the ground state minimum geometry were labeled according to their electronic character and the states at all other geometries were assigned based on their overlaps with the reference states (Fig. 4). From this, we can confirm that the main contribution to the first band in the spectrum of pyrrole is from the $\mathrm{B}_{2}\left(\pi \pi^{\star}\right)$ state which also has the largest oscillator strength. However, we also see significant contributions to the spectrum, especially at lower energies, from states with primarily $\mathrm{B}_{1}\left(\pi \sigma^{\star}\right), \mathrm{A}_{2}\left(3 p_{z}\right)$ or $\mathrm{B}_{1}\left(3 p_{y}\right)$ character which have negligible os- 
cillator strengths at the ground state minimum geometry. Looking at these states at other geometries (Fig. 4b and 4c), it can be seen that their oscillator strengths are proportional to their overlaps with the $\mathrm{B}_{2}^{\mathrm{vert}}\left(\pi \pi^{\star}\right)$ state at the ground state minimum geometry (where it is the $S_{5}$ state), indicating that their contributions to the spectrum are due to their mixing with the bright $\mathrm{B}_{2}\left(\pi \pi^{\star}\right)$ state. This qualitative analysis agrees well with the spectrum of pyrrole calculated from wavepacket propagations using MCTDH which have shown that intensity borrowing from the $\mathrm{B}_{2}\left(\pi \pi^{\star}\right)$ to the lower-lying states plays a significant role. ${ }^{56}$
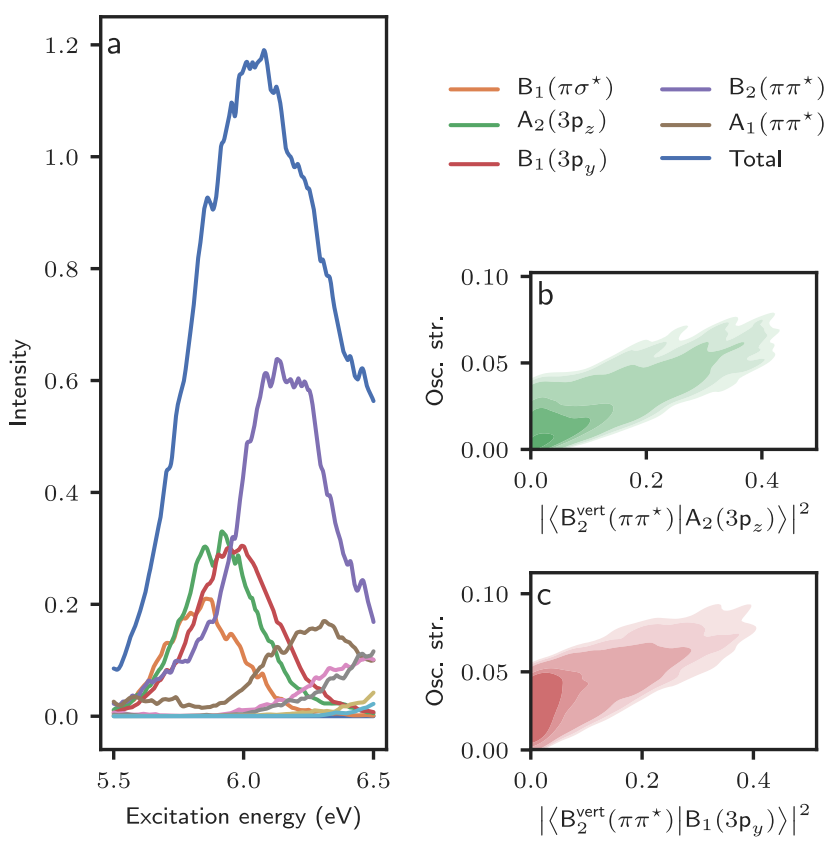

Figure 4: (a) Spectrum of pyrrole computed using the nuclear ensemble method and decomposed into contributions by state based on overlaps with the reference states defined at the ground state minimum geometry. Evidence of intensity borrowing from the $\mathrm{B}_{2}\left(\pi \pi^{\star}\right)$ to the (b) $\mathrm{A}_{2}\left(3 p_{z}\right)$ and (c) $\mathrm{B}_{1}\left(3 p_{y}\right)$ states can be seen by examining the dependence of the oscillator strength of those states to their overlaps with the $\mathrm{B}_{2}\left(\pi \pi^{\star}\right)$ state at the ground state minimum geometry.

Next, we focus on the geometry optimization of the bright $\mathrm{B}_{2}\left(\pi \pi^{\star}\right)$ state (Fig 5$)$. To locate the $\mathrm{B}_{2}\left(\pi \pi^{\star}\right)$ minimum, we start the optimization from the $S_{5}$ state at the ground state minimum geometry and, at each subsequent step, we continue the optimization on the adiabatic state which is characterized as the $\mathrm{B}_{2}\left(\pi \pi^{\star}\right)$ state at the current geometry (i.e. mapped to the $S_{5}$ state at the initial geometry), based on the overlap matrix assignment. 
In this way, when a conical intersection is reached during optimization, the optimization continues on the target state, unlike the standard adiabatic state geometry optimization, which usually reaches the first CI and fails to converge. In the present example, three such conical intersections (with the $\mathrm{B}_{1}\left(\pi \sigma^{\star}\right), \mathrm{B}_{1}\left(3 \mathrm{p}_{\mathrm{y}}\right)$ and $\mathrm{A}_{2}\left(3 \mathrm{p}_{\mathrm{z}}\right)$ states) are crossed and the algorithm converges to a minimum where $\mathrm{B}_{2}\left(\pi \pi^{\star}\right)$ is the $S_{2}$ adiabatic state. From the dominant NTO pairs of the target state along the optimization path (Fig. 5c), it is obvious that the electronic character of the state remained unchanged during optimization. The $\mathrm{B}_{2}\left(\pi \pi^{\star}\right)$ minimum, reported previously, ${ }^{40,61}$ is non-planar with the $\mathrm{N}$ atom and $\mathrm{H}$ atoms out of plane in opposite directions. Relaxation towards this minimum is the initial step of the main deactivation pathway of pyrrole upon excitation at $\approx 200 \mathrm{~nm} .{ }^{40}$

a

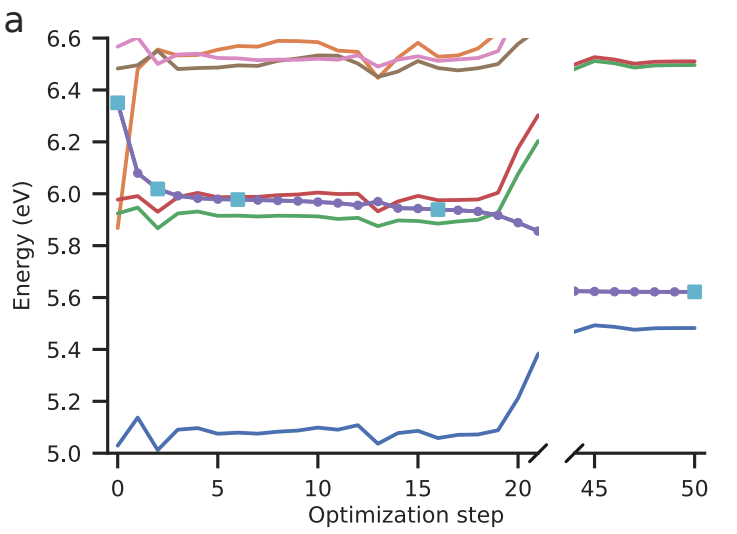

C

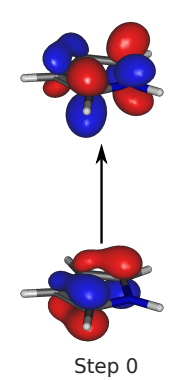

b

$\mathrm{B}_{2}\left(\pi \sigma^{*}\right)$ minimum

energy structure
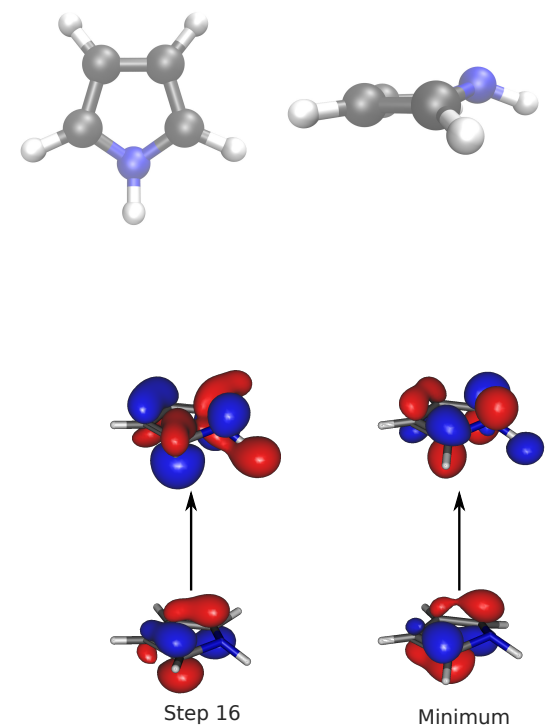

Figure 5: (a) Optimization of the $\mathrm{B}_{2}\left(\pi \pi^{\star}\right)$ state of pyrrole at the B3LYP/def2-TZVPD level of theory with three CI crossings occurring during the first 20 steps. (b) The converged minimum energy structure. (c) The dominant natural transition orbital pairs for the target state along the optimization path (at points marked by light blue squares)

The presented algorithm can simplify initial explorations of potential energy surfaces as 
it gives a rough guess of the motion along the initially populated diabatic state. It gives the location of the minimum, but also approximate CI structures which can be optimized to locate minimum energy CIs or used as starting points for optimizations of other diabatic states. These physically relevant geometries can provide insight into the PES, but can also be used to assess the applicability of the chosen electronic structure method as vertical excitation energies are not an adequate indicator of the quality of the results with a given method. Let us mention, however, that the algorithm can fail to converge if there is heavy mixing between adiabatic states in a wide PES region making the one to one assignment between states at different geometries meaningless. Also, when the reference states are fixed at the initial geometry, the overlaps systematically become smaller as the geometry moves further from the initial guess. This is mostly recovered by orthogonalization of the overlap matrix since all overlaps are affected by the translation of the basis set in the same way, but changes to the algorithm would be required for large displacements from the initial geometry.

\section{Conclusion}

A code for calculating overlap matrices between CIS type wave functions has been developed using two very efficient new algorithms, OL2M and ONTO, based on, respectively, a two step Laplace's recursive formula and expansion of the wave functions into natural transition orbitals. Overall, the ONTO algorithm allows us to calculate the overlap matrix elements without any approximations for all systems typically investigated using FSSH approaches with the LR-TDDFT or ADC(2) methods, shifting the bottleneck of such calculations back to the electronic structure method without making any approximations to the overlaps. For specific situations where one might need a very large number of states in the calculation, the L2M algorithm is preferred. Additionally, we show how even seemingly small approximations to the wave function can result in large errors in the calculated overlaps, which further 
demonstrates the need for efficient calculations of exact wave function overlaps. The code is parallelized and has been interfaced to the Turbomole program package, but can easily be interfaced to any electronic structure program.

Nonadiabatic dynamics simulations are the most common use of wave function overlaps. However, there are many possible applications and extensions of the present algorithms. Using pyrrole as an illustrative example, we show the utility of the overlap calculation for the assignment of UV absorption spectra obtained by sampling over a large number of nuclear geometries or for following the electronic character of a state along a path in nuclear coordinate space. Other applications include comparison of results obtained with different electronic structure methods or basis sets, ${ }^{42}$ or calculation of Dyson orbitals for the simulation of time resolved photoelectron spectra. ${ }^{19}$ Further work is in progress along these lines.

\section{Acknowledgement}

This research has been supported by the Croatian Science Fundation under the grant HRZZ IP-2016-06-1142 and by the European Regional Development Fund under the grant KK.01.1.1.01.0009 (DATACROSS) and by the Foundation of the Croatian Academy of Science and Arts. Parts of this research were performed using the resources of computer cluster Isabella based in SRCE - University of Zagreb University Computing Centre.

\section{References}

(1) Cimiraglia, R.; Malrieu, J.-P.; Persico, M.; Spiegelmann, F. Journal of Physics B: Atomic and Molecular Physics 1985, 18, 3073-3084.

(2) Durand, P.; Malrieu, J.-P. In Advances in Chemical Physics; Lawley, K. P., Ed.; John Wiley \& Sons, Ltd, 1987; Vol. 67; pp 321-412. 
(3) Pacher, T.; Cederbaum, L. S.; Köppel, H. The Journal of Chemical Physics 1988, 89, $7367-7381$.

(4) Pacher, T.; Köppel, H.; Cederbaum, L. S. The Journal of Chemical Physics 1991, 95, $6668-6680$.

(5) Domcke, W.; Woywod, C. Chemical Physics Letters 1993, 216, 362-368.

(6) Domcke, W.; Woywod, C.; Stengle, M. Chemical Physics Letters 1994, 226, 257-262.

(7) Venghaus, F.; Eisfeld, W. The Journal of Chemical Physics 2016, 144, 114110.

(8) Gadéa, F. X.; Pélissier, M. The Journal of Chemical Physics 1990, 93, 545-551.

(9) Ruedenberg, K.; Atchity, G. J. The Journal of Chemical Physics 1993, 99, 3799-3803.

(10) Atchity, G. J.; Ruedenberg, K. Theoretical Chemistry Accounts: Theory, Computation, and Modeling (Theoretica Chimica Acta) 1997, 97, 47-58.

(11) Nakamura, H.; Truhlar, D. G. Journal of Chemical Physics 2001, 115, 10353-10372.

(12) Nakamura, H.; Truhlar, D. G. Journal of Chemical Physics 2002, 117, 5576-5593.

(13) Yang, K. R.; Xu, X.; Truhlar, D. G. Chemical Physics Letters 2013, 573, 84-89.

(14) Li, S. L.; Truhlar, D. G.; Schmidt, M. W.; Gordon, M. S. The Journal of Chemical Physics 2015, 142, 064106.

(15) Thürwächter, R.; Halvick, P. Chemical Physics 1997, 221, 33-44.

(16) Oana, C. M.; Krylov, A. I. The Journal of Chemical Physics 2007, 127, 234106.

(17) Oana, C. M.; Krylov, A. I. Journal of Chemical Physics 2009, 131.

(18) Walter, M.; Häkkinen, H. New Journal of Physics 2008, 10, 043018. 
(19) Humeniuk, A.; Wohlgemuth, M.; Suzuki, T.; Mitrić, R. The Journal of Chemical Physics 2013, 139, 134104.

(20) Ponzi, A.; Angeli, C.; Cimiraglia, R.; Coriani, S.; Decleva, P. The Journal of Chemical Physics 2014, 140, 204304.

(21) Ponzi, A.; Sapunar, M.; Angeli, C.; Cimiraglia, R.; Došlić, N.; Decleva, P. The Journal of Chemical Physics 2016, 144, 084307.

(22) Tully, J. C. The Journal of Chemical Physics 1990, 93, 1061-1071.

(23) Hammes-Schiffer, S.; Tully, J. C. The Journal of Chemical Physics 1994, 101, 46574667.

(24) Fabiano, E.; Keal, T. W.; Thiel, W. Chemical Physics 2008, 349, 334-347.

(25) Pittner, J.; Lischka, H.; Barbatti, M. Chemical Physics 2009, 356, 147-152.

(26) Granucci, G.; Persico, M.; Toniolo, A. The Journal of Chemical Physics 2001, 114, $10608-10615$.

(27) Fernandez-Alberti, S.; Roitberg, A. E.; Nelson, T.; Tretiak, S. The Journal of Chemical Physics 2012, 137, 014512.

(28) Wang, L.; Prezhdo, O. V. Journal of Physical Chemistry Letters 2014, 5, 713-719.

(29) Qiu, J.; Bai, X.; Wang, L. The Journal of Physical Chemistry Letters 2018, 9, 43194325.

(30) Meek, G. A.; Levine, B. G. Journal of Physical Chemistry Letters 2014, 5, 2351-2356.

(31) Plasser, F.; Granucci, G.; Pittner, J.; Barbatti, M.; Persico, M.; Lischka, H. The Journal of Chemical Physics 2012, 13\%, 22A514. 
(32) Nelson, T.; Fernandez-Alberti, S.; Roitberg, A. E.; Tretiak, S. Chemical Physics Letters 2013, 590, 208-213.

(33) Ryabinkin, I. G.; Nagesh, J.; Izmaylov, A. F. The Journal of Physical Chemistry Letters 2015, 6, 4200-4203.

(34) Schirmer, J. Physical Review A 1982, 26, 2395-2416.

(35) Dreuw, A.; Wormit, M. Wiley Interdisciplinary Reviews: Computational Molecular Science 2015, 5, 82-95.

(36) Casida, M. E. In Recent Advances in Density Functional Methods; Chong, D. P., Ed.; World Scientific: Singapore, 1995; pp 155-192.

(37) Tapavicza, E.; Tavernelli, I.; Rothlisberger, U. Physical Review Letters 2007, 98, 023001.

(38) Werner, U.; Mitrić, R.; Suzuki, T.; Bonačić-Koutecký, V. Chemical Physics 2008, 349, $319-324$.

(39) Plasser, F.; Crespo-Otero, R.; Pederzoli, M.; Pittner, J.; Lischka, H.; Barbatti, M. Journal of Chemical Theory and Computation 2014, 10, 1395-1405.

(40) Sapunar, M.; Ponzi, A.; Chaiwongwattana, S.; Mališ, M.; Prlj, A.; Decleva, P.; Došlić, N. Physical Chemistry Chemical Physics 2015, 17, 19012-19020.

(41) Löwdin, P. O. Physical Review 1955, 97, 1474-1489.

(42) Plasser, F.; Ruckenbauer, M.; Mai, S.; Oppel, M.; Marquetand, P.; González, L. Journal of Chemical Theory and Computation 2016, 12, 1207-1219.

(43) Martin, R. L. Journal of Chemical Physics 2003, 118, 4775-4777.

(44) Mayer, I. Chemical Physics Letters 2007, 437, 284-286. 
(45) Surján, P. R. Chemical Physics Letters 2007, 439, 393-394.

(46) Furche, F.; Ahlrichs, R.; Hättig, C.; Klopper, W.; Sierka, M.; Weigend, F. Wiley Interdisciplinary Reviews: Computational Molecular Science 2014, 4, 91-100.

(47) Furche, F.; Ahlrichs, R. Journal of Chemical Physics 2002, 117, 7433-7447.

(48) Hättig, C. Advances in Quantum Chemistry; 2005; Vol. 50; pp 37-60.

(49) Munkres, J. Journal of the Society for Industrial and Applied Mathematics 1957, 5, $32-38$.

(50) Vallet, V.; Lan, Z.; Mahapatra, S.; Sobolewski, A. L.; Domcke, W. Faraday Discuss. 2004, 127, 283-293.

(51) Vallet, V.; Lan, Z.; Mahapatra, S.; Sobolewski, A. L.; Domcke, W. The Journal of Chemical Physics 2005, 123, 144307.

(52) Vazdar, M.; Eckert-Maksić, M.; Barbatti, M.; Lischka, H. Molecular Physics 2009, 107, $845-854$.

(53) Barbatti, M.; Pittner, J.; Pederzoli, M.; Werner, U.; Mitrić, R.; Bonačić-Koutecký, V.; Lischka, H. Chemical Physics 2010, 375, 26-34.

(54) Roberts, G. M.; Williams, C. A.; Yu, H.; Chatterley, A. S.; Young, J. D.; Ullrich, S.; Stavros, V. G. Faraday Discussions 2013, 163, 95.

(55) Karsili, T. N. V.; Marchetti, B.; Moca, R.; Ashfold, M. N. R. The Journal of Physical Chemistry A 2013, 117, 12067-12074.

(56) Neville, S. P.; Worth, G. A. The Journal of Chemical Physics 2014, 140, 034317.

(57) Wu, G.; Neville, S. P.; Schalk, O.; Sekikawa, T.; Ashfold, M. N. R.; Worth, G. A.; Stolow, A. The Journal of Chemical Physics 2015, 142, 074302. 
(58) Barbatti, M.; Aquino, A. J. A.; Lischka, H. Physical Chemistry Chemical Physics 2010, $12,4959$.

(59) Crespo-Otero, R.; Barbatti, M. Theoretical Chemistry Accounts 2012, 131, 1-14.

(60) Barbatti, M.; Sen, K. International Journal of Quantum Chemistry 2016, 116, 762-771.

(61) Celani, P.; Werner, H.-J. The Journal of Chemical Physics 2003, 119, 5044-5057. 


\section{Graphical TOC Entry}

